\title{
Circular RNA HIPK3: an emerging player in diabetes
}

\author{
Xiaoyun $\mathrm{He}^{1,2,3}$, Chibin $\mathrm{Pu}^{4}$, Yongsheng Quan ${ }^{5}$, Chunlin Ou ${ }^{1 \#}$, Suxian Zhou ${ }^{2,3 \#}$ \\ ${ }^{1}$ Department of Pathology, ${ }^{2}$ Department of Endocrinology, Xiangya Hospital, Central South University, Changsha 410008, China; ${ }^{3}$ Department \\ of Endocrinology, Affiliated Hospital of Guilin Medical University, Guilin 541001, China; ${ }^{4}$ Department of Gastroenterology, Zhongda Hospital, \\ Southeast University, Nanjing 210009, China; ${ }^{5}$ Department of Emergency, Xiangya Hospital, Central South University, Changsha 410008, China \\ "These authors jointly supervised this work. \\ Correspondence to: Chunlin Ou. Department of Pathology, Xiangya Hospital, Central South University, 87 Xiangya Road, Changsha 410008, China. \\ Email: ouchunlin@csu.edu.cn; Suxian Zhou. Department of Endocrinology, Xiangya Hospital, Central South University, 87 Xiangya Road, Changsha \\ 410008, China. Email: 574569504@qq.com. \\ Comment on: Shan K, Liu C, Liu B, et al. Circular Noncoding RNA HIPK3 Mediates Retinal Vascular Dysfunction in Diabetes Mellitus. Circulation \\ 2017;136:1629-42.
}

Submitted May 08, 2018. Accepted for publication May 25, 2018.

doi: $10.21037 /$ tcr.2018.05.46

View this article at: http://dx.doi.org/10.21037/tcr.2018.05.46

With rapid global aging, the occurrence of diabetes mellitus (DM) and its associated complications, including diabetic retinopathy (DR), is also increasing. DR is one of the major complications of DM and a leading cause of visual impairment in developed countries (1). DR-induced visual impairment is usually accompanied by vascular hyperpermeability, inflammation, neovascularization, retinal ischemia, and vascular cell dysfunction (2-4), and it has become a primary cause leading to blindness. DR is characterized by abnormalities in either the structure or functionality of the retinal microvasculature (5). Due to the different proliferation statuses of retinal neovascularization, DR can be divided into proliferative diabetic retinopathy (PDR) and non-proliferative diabetic retinopathy (NPDR) $(6,7)$ There are many hypotheses about the pathogenesis of DR. The most prominent one being that persistent hyperglycemia causes the formation of glycation end products, which activates the hexosamine pathway, which in turn triggers the apoptosis of retinal neurons. In addition, genetic susceptibility, immune response, inflammation, and disease have also been implicated in the onset of DR. DR has notable clinical and pathological features, and identifying biomarkers that can predict DR or therapeutic efficacy is crucial because of its complex pathogenesis and indistinct risk factors $(8,9)$. With the accumulation of scientific data, the diagnosis and treatment of DR have improved significantly. However, there has been no substantial reduction in DR-related mortality. Therefore, an effective treatment to improve the long-term survival and life quality of DR patients is urgently needed.

Non-coding RNAs (ncRNAs) include microRNAs (miRNAs) (10), long non-coding RNAs (lncRNAs) $(11,12)$, and circular RNAs (circRNAs) (13). CircRNAs are mainly derived from exons or introns, which are generated by two kinds of splicing-exons reverse splicing of exons and introns (14). Unlike linear RNAs, circRNAs have neither 5' or 3' polarity nor a polyadenylate tail. They are closed loop structures that are not affected by RNA exonucleases, which makes them highly stable and non-degradable, and are abundant in the cytoplasm of eukaryotic cells. CircRNAs can function as a miRNA sponges to regulate miRNA activity by competitively binding to miRNA target sites, thereby decreasing or inhibiting their function and ultimately affecting the expression of the miRNA target genes $(15,16)$. Recent evidence shows that circRNAs play a role in the occurrence and development of diabetes, atherosclerosis, nervous system disorders, cancers, and other diseases $(17,18)$. Yang et al. (19) reported that the circRNA Circ-FBXW7 can translate proteins and plays an important role in glioma. This was the first report of a protein translated by a circRNA working synergistically with the protein expression products of the parent gene. Zeng et al. (20) found that circ-Amolt1 is highly expressed in the neonatal heart, where it promotes the phosphorylation and nuclear import of Akt1 through interaction with PDK1 and AKT1. This mechanism was further confirmed in cells in 
vitro as well as in an animal model of doxorubicin-induced cardiomyopathy. Moreover, Zhang et al. (21) demonstrated that circ-LARP4 can inhibit miR-424-5p competitively and regulate the LATS1 gene in the Hippo pathway, thereby contributing to the development of gastric cancer. These findings suggest that circRNAs could be robust molecular markers of diseases. An in-depth understanding of the mechanisms and functions of circRNAs will thus provide valuable insights into disease development, as well as identifying potential biological markers for disease diagnosis and prediction of therapeutic efficacy.

A recent article in Circulation by Shan et al. (22) suggests a novel role of circRNAs in the progression and development of DR. In this study, the authors explored the role of circRNA in retinal vascular dysfunction caused by diabetes and found that circ-HIPK 3 is significantly elevated in diabetic retinal vascular disorders. Silencing or overexpression of circ-HIPK3 dysregulated the viability, proliferation, migration, and tube formation ability of retinal endothelial cells. Investigation of the molecular mechanism revealed that circ-HIPK 3 competitively bound miR-30a-3p, miR-30d-3p, and miR-30e-3p to reverse the expression of their respective target genes, VEGF, FZD4, and WNT2, thus forming a new regulatory network comprising circRNAs-miRNAs-mRNAs that plays an important role in diabetic retinal vascular dysfunction. However, I consider the experimental design described for Figure $3 \mathrm{D}$ is flawed. The authors should design two categories of 3'-biotinylated miRNA duplexes: wild-type and one with a mutation in the circ-HIPK3 binding site. This investigation would powerfully support the conclusion of circ-HIPK3 sponging miR-30a-3p, miR-30d-3p, miR30e-3p.

In brief, Shan et al. (22) clearly clarified the regulatory mechanism of the novel circRNA HIPK3 in diabetic proliferative retinopathy and provided a potential strategy for the therapeutic targeting of circ-HIPK3 in DR. CircRNA research has become more systematic and standardized with the rapid development of molecular biological information technology and in-depth RNA sequencing technology. The three most frequently used research tools in this field are molecular biological methods, genomic methods, and circRNA-specific databases. The circRNA-specific databases, including circ Base, circ2Traits, and circ Net, contain information on nearly 100,000 circRNA sequences and can predict circRNA-miRNAmRNA interactions, which is invaluable for the systematic study of circRNAs. Thus, circRNAs have become novel diagnostic biomarkers and therapeutic targets for DR. However, identification and validation of novel functional circRNAs in vivo and in vitro are needed to explore the potential molecular mechanisms of diseases. Although accumulating evidence clearly supports the potential value of circRNAs in diagnosis and therapeutics of various diseases, their clinical applications remain unknown. It is believed that with the shift of research on circRNA and disease from the "hypothesis" stage to the "practice proof" stage, circRNAs will bring more surprises and breakthroughs for the medical and scientific communities.

\section{Acknowledgments}

Funding: This work was supported by the Fundamental Research Funds for the Central Universities of Central South University (2018zzts044).

\section{Footnote}

Provenance and Peer Review: This article was commissioned and reviewed by the Section Editor Chunlin Ou (Cancer Research Institute of Central South University, Changsha, China).

Conflicts of Interest: All authors have completed the ICMJE uniform disclosure form (available at http://dx.doi. org/10.21037/tcr.2018.05.46). The authors have no conflicts of interest to declare.

Ethical Statement: The authors are accountable for all aspects of the work in ensuring that questions related to the accuracy or integrity of any part of the work are appropriately investigated and resolved.

Open Access Statement: This is an Open Access article distributed in accordance with the Creative Commons Attribution-NonCommercial-NoDerivs 4.0 International License (CC BY-NC-ND 4.0), which permits the noncommercial replication and distribution of the article with the strict proviso that no changes or edits are made and the original work is properly cited (including links to both the formal publication through the relevant DOI and the license). See: https://creativecommons.org/licenses/by-nc-nd/4.0/.

\section{References}

1. Yau JW, Rogers SL, Kawasaki R, et al. Global prevalence 
and major risk factors of diabetic retinopathy. Diabetes Care 2012;35:556-64.

2. Brownlee M. Biochemistry and molecular cell biology of diabetic complications. Nature 2001;414:813-20.

3. Ou C, Sun Z, Zhang H, et al. SPLUNC1 reduces the inflammatory response of nasopharyngeal carcinoma cells infected with the EB virus by inhibiting the TLR9/NFkappaB pathway. Oncol Rep 2015;33:2779-88.

4. Zhou SX, Huo DM, He XY, et al. High glucose/ lysophosphatidylcholine levels stimulate extracellular matrix deposition in diabetic nephropathy via plateletactivating factor receptor. Mol Med Rep 2018;17:2366-72.

5. Michalik KM, You X, Manavski Y, et al. Long noncoding RNA MALAT1 regulates endothelial cell function and vessel growth. Circ Res 2014;114:1389-97.

6. Curtis TM, Gardiner TA, Stitt AW. Microvascular lesions of diabetic retinopathy: clues towards understanding pathogenesis? Eye (Lond) 2009;23:1496-508.

7. Wu L, Fernandez-Loaiza P, Sauma J, et al. Classification of diabetic retinopathy and diabetic macular edema. World J Diabetes 2013;4:290-4.

8. Ting DS, Tan KA, Phua V, et al. Biomarkers of Diabetic Retinopathy. Curr Diab Rep 2016;16:125.

9. Dhoot DS, Avery RL. Vascular Endothelial Growth Factor Inhibitors for Diabetic Retinopathy. Curr Diab Rep 2016;16:122.

10. Ou C, Sun Z, Li X, et al. MiR-590-5p, a density-sensitive microRNA, inhibits tumorigenesis by targeting YAP1 in colorectal cancer. Cancer Lett 2017;399:53-63.

11. He X, Ou C, Xiao Y, et al. LncRNAs: key players and novel insights into diabetes mellitus. Oncotarget 2017;8:71325-41.

12. Ou C, Li G. Long non-coding RNA TUG1: a novel therapeutic target in small cell lung cancer. J Thorac Dis
2017;9:E644-5.

13. He X, Kuang G, Ou C, et al. Crosstalk between circular RNAs and microRNAs in tumorigenesis. Transl Cancer Res 2017;6:S1448-50.

14. Li J, Yang J, Zhou P, et al. Circular RNAs in cancer: novel insights into origins, properties, functions and implications. Am J Cancer Res 2015:5:472-80.

15. Memczak S, Jens M, Elefsinioti A, et al. Circular RNAs are a large class of animal RNAs with regulatory potency. Nature 2013;495:333-8.

16. Hansen TB, Jensen TI, Clausen BH, et al. Natural RNA circles function as efficient microRNA sponges. Nature 2013;495:384-8.

17. Burd CE, Jeck WR, Liu Y, et al. Expression of linear and novel circular forms of an INK4/ARF-associated noncoding RNA correlates with atherosclerosis risk. PLoS Genet 2010;6:e1001233.

18. Li F, Zhang L, Li W, et al. Circular RNA ITCH has inhibitory effect on ESCC by suppressing the Wnt/betacatenin pathway. Oncotarget 2015;6:6001-13.

19. Yang Y, Gao X, Zhang M, et al. Novel Role of FBXW7 Circular RNA in Repressing Glioma Tumorigenesis. J Natl Cancer Inst 2018;110. doi: 10.1093/jnci/djx166.

20. Zeng Y, Du WW, Wu Y, et al. A Circular RNA Binds To and Activates AKT Phosphorylation and Nuclear Localization Reducing Apoptosis and Enhancing Cardiac Repair. Theranostics 2017;7:3842-55.

21. Zhang J, Liu H, Hou L, et al. Circular RNA_LARP4 inhibits cell proliferation and invasion of gastric cancer by sponging miR-424-5p and regulating LATS1 expression. Mol Cancer 2017;16:151.

22. Shan K, Liu C, Liu BH, et al. Circular Noncoding RNA HIPK3 Mediates Retinal Vascular Dysfunction in Diabetes Mellitus. Circulation 2017;136:1629-42.
Cite this article as: $\mathrm{He} \mathrm{X,} \mathrm{Pu} \mathrm{C,} \mathrm{Quan} \mathrm{Y,} \mathrm{Ou} \mathrm{C,} \mathrm{Zhou} \mathrm{S.}$ Circular RNA HIPK3: an emerging player in diabetes. Transl Cancer Res 2018;7(Suppl 7):S715-S717. doi: 10.21037/ tcr.2018.05.46 